\title{
Cerebellar Substrates for Error Correction in Motor Conditioning
}

\author{
Mark A. Gluck,* M. Todd Allen,* Catherine E. Myers, $†$ and Richard F. Thompson $\ddagger$ \\ *Center for Molecular and Behavioral Neuroscience, Rutgers-Newark; †Department of Psychology, \\ Rutgers-Newark; and $\ddagger$ Neuroscience Program, University of Southern California
}

\begin{abstract}
The authors evaluate a mapping of Rescorla and Wagner's (1972) behavioral model of classical conditioning onto the cerebellar substrates for motor reflex learning and illustrate how the limitations of the Rescorla-Wagner model are just as useful as its successes for guiding the development of new psychobiological theories of learning. They postulate that the inhibitory pathway that returns conditioned response information from the cerebellar interpositus nucleus back to the inferior olive is the neural basis for the error correction learning proposed by Rescorla and Wagner (Gluck, Myers, \& Thompson, 1994; Thompson, 1986). The authors' cerebellar model expects that behavioral processes described by the Rescorla-Wagner model will be localized within the cerebellum and related brain stem structures, whereas behavioral processes beyond the scope of the Rescorla-Wagner model will depend on extracerebellar structures such as the hippocampus and related cortical regions. Simulations presented here support both implications. Several novel implications of the authors' cerebellar error-correcting model are described including a recent empirical study by Kim, Krupa, and Thompson (1998), who verified that suppressing the putative error correction pathway should interfere with the Kamin (1969) blocking effect, a behavioral manifestation of error correction learning. The authors also discuss the model's implications for understanding the limits of cerebellar contributions to associative learning and how this informs our understanding of hippocampal function in conditioning. This leads to a more integrative view of the neural substrates of conditioning in which the authors' realtime circuit-level model of the cerebellum can be viewed as a generalization of the long-term memory module of Gluck and Myers' (1993) trial-level theory of cerebellar-hippocampal interaction in motor conditioning. (๑) 2001 Academic Press
\end{abstract}

For their thoughtful comments and advice on this work, we are indebted to Richard Granger, James Kehoe, and Joe Steinmetz. This research was supported by the Office of Naval Research (ONR) by a grant to M.G. (N00014-88-K-0112) as well as by an ONR Young Investigator Award, a National Science Foundation (NSF) Presidential Early Career Award, and a grant from the New Jersey Governor's Council on Autism to M.G.; by a National Institute of Mental Health (NIMH) National Research Service Award (1-F32-MH10351-01) to C.M.; and by grants to R.T. from the NSF (BN9215069), ONR (N00014-95-1), NIMH (P20-MH52194), National Institute on Aging (AG14751), and Sankyo Corporation.

Address correspondence and reprint requests to Mark A. Gluck, Center for Molecular and Behavioral Neuroscience, Rutgers University, 197 University Ave., Newark, NJ 07102. E-mail: gluck@pavlov.rutgers.edu. 
Because of the high degree of experimental control possible, classical conditioning has proven to be an especially valuable paradigm for the analysis of the brain substrates of associative learning. One of the best characterized learning preparations is classical eyeblink conditioning (Fig. 1A), which has been studied in both humans (Woodruff-Pak, 1999) and animals, especially rabbits (for reviews, see Anderson \& Steinmetz, 1994; Gormezano, Kehoe, \& Marshall, 1983). This learning is based on a naturally occurring motor reflex response: eyelid closure in response to a corneal air puff or periorbital shock (the unconditioned stimulus [US]), as shown in Fig. 1B. If the US is repeatedly preceded by a neutral stimulus such as a tone or a light (the conditioned stimulus [CS]), then the CS itself comes to evoke an anticipatory, well-timed, protective eyeblink (the conditioned response $[\mathrm{CR}]$ ). This simple learning paradigm can be expanded to include multiple CSs or USs, temporal manipulations, and contextual influences (Gormezano et al., 1983).

Work by Richard Thompson and colleagues over the past two decades has pointed to the cerebellum as the site for the essential memory traces for motor conditioning in vertebrates (for reviews, see Kim et al., 1998; Steinmetz \& Thompson, 1991). Building on this empirical progress, we have shown in a series of theoretical modeling articles over the past 10 years how psychological theories of animal learning can lead to a deeper understanding of the functional role of specific components of these cerebellar circuits (Donegan, Gluck, \& Thompson, 1989; Gluck, Myers, \& Thompson, 1994; Gluck, Reifsnider, \& Thompson, 1990).

An essential cornerstone of our psychobiological theories is the behavioral model of conditioning proposed in 1972 by Robert Rescorla and Alan Wagner (Rescorla \& Wagner,

\section{(A)}

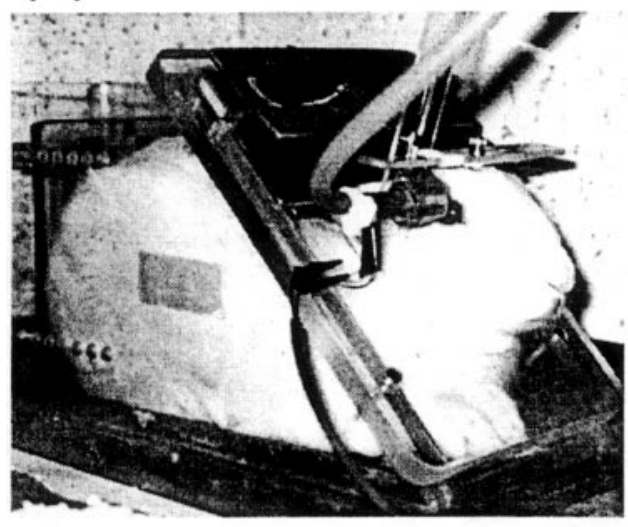

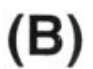

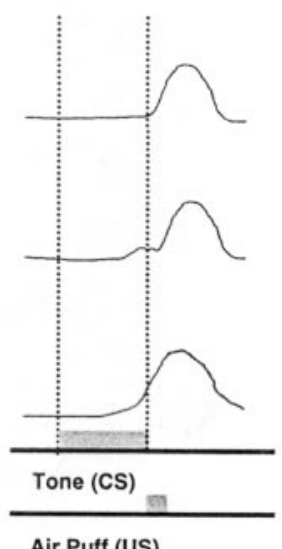

Day 1:

Eyeblink UR

Day 3:

Eyeblink CR / UR

Day 5:

Eyeblink CR / UR

FIG. 1. Rabbit eyeblink conditioning apparatus and behavioral responses recorded across training. (A) Rabbit eyeblink apparatus with a rabbit in the standard restraint box in the conditioning chamber. The tone CS is administered via a speaker (not shown). The corneal air puff US is administered via the air hose (shown). The behavioral eyeblink response is monitored by an infrared detector aimed at the animal's eye. (B) Development of the conditioned eyeblink response. On day 1, only reflexive URs are exhibited following the air puff US presentation. There is no initial eyeblink response to the CS tone. On day 3, small CRs that precede the US are exhibited. On day 5, large CRs are exhibited that peak at about the time of US presentation. Not only does the rabbit learn that the tone predicts the air puff, it also learns the precise temporal relationship between the stimuli. 
1972; Wagner \& Rescorla, 1972). The Rescorla-Wagner model is a mathematical formulation of conditioning that assumes that the change in association between a neutral conditioned stimulus and a response-evoking unconditioned stimulus is a function of the difference between the US and an animal's expectation of the US given all CSs present on the trial. Because the discrepancy, or "error," between the animals' expectations and what actually occurs drives learning in this theory, the theory is referred to as an "errorcorrecting" learning procedure.

Nearly 25 years after its publication, the Rescorla-Wagner model still stands as the most influential and powerful model in psychology for describing and predicting animal learning behavior in conditioning studies (for reviews, see Miller, Barnet, \& Grahame, 1995; Walkenbach \& Haddad, 1980). Moreover, its influence has extended far beyond animal conditioning. The model's basic error correction principle has been rediscovered within cognitive psychology and cognitive neuroscience in the form of connectionist network models, which rely on the same principle (Gluck \& Bower, 1988). In addition, the most commonly used connectionist learning procedure, error back-propagation (Rumelhart, Hinton, \& Williams, 1986), along with its simpler predecessor, the leastmeans square rule (Widrow \& Hoff, 1960), are generalizations of the Rescorla-Wagner model.

Sutton and Barto (1981) proposed an adaptive network model of classical conditioning that used a form of expectation closely related to the Rescorla-Wagner rule. The Sutton and Barto adaptive model incorporates the predictive nature of classical conditioning. Whereas the Rescorla-Wagner rule generated an expectation of the US based on a summation of all the stimuli present during a trial, the Sutton and Barto model made distinctions between stimuli based on their time of occurrence relative to the US. The Sutton and Barto model was able to expand on the Rescorla-Wagner rule by identifying stimuli that are reliable, nonredundant, and early predictors of the US, not just reliable nonredundant stimuli that are correlated with the US. This early prediction allowed the Sutton and Barto adaptive model to make predictions that precede the appearance of the US. The prediction that precedes the appearance of the US is the exact type of mechanism that has to occur in the brain so that neural activity changes can occur before, and initiate the conditioned behavioral response prior to the delivery of, the US. This is the type of mechanism that we describe in our model of the neural substrates of classical conditioning.

In several articles over the past decade, we have argued that the Rescorla-Wagner model can also be exploited to further our understanding of the neural substrates of classical conditioning (Donegan et al., 1989; Gluck et al., 1990, 1994). In this article, we illustrate how the limitations of the Rescorla-Wagner model are just as useful as its successes for guiding the development of new psychobiological theories of learning.

We describe here a simple computational instantiation of several aspects of the cerebellar circuitry for conditioning. This model is not intended as a full-scale detailed neurobiological model incorporating all that is known about the cerebellum and its role in conditioning; rather, we have used modeling as a tool to help us understand the functional role of two critical circuit pathways within the cerebellum. This reduced model of the cerebellum implements the essential error correction features of the Rescorla-Wagner theory and is sufficient to generate a range of behaviors similar to those mediated by the cerebellum. The initial qualitative idea behind this mapping of the Rescorla-Wagner learning rule onto cerebellar circuitry grew out of empirical and theoretical work in the Thompson 
laboratory at Stanford University during the early to mid-1980s with key contributions from Nelson Donegan, Mark Gluck, Joseph Steinmetz, and Richard Thompson. The basic qualitative idea was first presented in Donegan et al. (1989), and a preliminary computational model was described in a brief book chapter (Gluck et al., 1994). This article provides the first detailed description and analysis of the model along with a comparison of the model to other relevant theories and models of the cerebellar and hippocampal contributions to conditioning.

We also review here a recent empirical study by Kim et al. (1998) that validates and supports one of our model's novel predictions. We discuss the model's implications for understanding the limits of cerebellar contributions to associative learning and how this informs our understanding of the contribution of the hippocampal region to stimulus selection in conditioning. This leads to a more integrative view of the neural substrates of conditioning in which this real-time circuit-level model of the cerebellum can be viewed as a generalization of the long-term memory module of Gluck and Myers' (1993) more abstract trial-level model of cerebellar-hippocampal interaction in motor reflex conditioning. In the next section, we review some of the psychological theories of learning that form the framework for our current psychobiological modeling.

\section{ERROR CORRECTION LEARNING IN CLASSICAL CONDITIONING}

Early psychological theories of learning assumed that the co-occurrence of a CS and a US was, by itself, sufficient to produce associative learning (Hull, 1943; Spence, 1956). Within neuroscience, similar principles underlie the Hebbian rule of associative learning, which proposes that associative weights between neurons will be incremented when one input signal co-occurs with another input signal (Hebb, 1949). The key implication of this Hebbian rule is that associative changes should be influenced only by the pairwise relationship between two signals. Thus, early theories of associative learning in both psychology and neuroscience presumed that associative changes are independent of the stimulus context in which signals occur.

During the late 1960s, however, several behavioral experiments in animal learning challenged this prevailing view that stimulus co-occurrence, known as stimulus "contiguity," was sufficient for learning. The most influential of these studies was Kamin's (1969) study of blocking in which rats first received pairings of a neutral tone CS with a painful foot shock US. Eventually, tone CS presentation produced a CR: suppression of feeding behaviors. The rats were then given trials with the stimulus compound, tone plus light, paired with the same shock US. If we refer to the tone as CS $A$ and the light as CS $B$, then we can characterize the first phase of training as $A+$ training and the second phase as $A B+$ training, as shown in Table 1. A later test showed a continued high conditioned responding to the tone alone ( $A$ test) but virtually no responding to the light alone ( $B$ test). Apparently, prior conditioning of $A+$ had blocked learning about $B$. This contrasted with other animals in a control condition that had received only $A B+$ training trials without the earlier tone $\rightarrow$ shock pairings. These control animals did show appreciable CRs when tested with either cue alone.

Kamin's (1969) blocking study, along with other similar studies published around the same period, demonstrated that the ability of a CS to become associated with a US depends on whether or not the CS imparts reliable and nonredundant information about the expected 
TABLE 1

The Blocking Experiment

\begin{tabular}{llll}
\hline Group & \multicolumn{1}{c}{ Phase 1 } & \multicolumn{1}{c}{ Phase 2 } & \multicolumn{1}{c}{ Test } \\
\hline Experiment & Tone $\rightarrow$ Shock & Tone + Light $\rightarrow$ Shock & Light \\
& $(A+)$ & $(A B+)$ & $(B$ test $)$ \\
Control & No stimuli & Tone + Light $\rightarrow$ Shock & Light \\
& & $(A B+)$ & $(B$ test $)$ \\
\hline
\end{tabular}

Source. Kamin (1969).

occurrence of the US (Rescorla, 1968; Wagner, 1969). In a related study, Rescorla (1968) showed that CS-US associations are highly sensitive not just to the co-occurrence (contiguity) between the CS and the US but also to their contingency, that is, whether or not the US is more likely to occur given that the CS is present relative to its likelihood given no CS. Altogether, these results suggest that learning in classical conditioning is highly sensitive to the informational value of the CS for predicting the US.

\section{The Rescorla-Wagner Model}

Kamin (1969) suggested that one way in which animals could learn to be sensitive to the informational value of a cue would be to change associative relations only in response to a US that is surprising or unexpected given all of the cues present on a trial. Rescorla and Wagner took this principle of learning from surprise and formalized it as a mathematical model that described a rule or process for predicting changes in CS-US associations (Rescorla \& Wagner, 1972; Wagner \& Rescorla, 1972). In the Rescorla-Wagner model, the associative changes accruing on a trial between a CS and a US are proportional to the degree to which the US is unexpected given the associative strengths associated with all of the CS cues that are present on that trial. To formulate the relationship, let $V_{A}$ denote the strength of association between stimulus $A$ and the US. If $A$ is followed by the US, then the change in associative strength between $A$ and the US, $\Delta V_{A}$, can be described by Eq. (1):

$$
\Delta V_{A}=\alpha_{A} \beta_{\uparrow}\left(\lambda-\sum_{k \in s} V_{k}\right),
$$

where $\alpha_{A}$ reflects the intensity or salience of $A, \beta \uparrow$ reflects the rate of learning on USpresent trials, $\lambda$ is the maximum possible level of associative strength conditionable with that US, and $\sum_{k \in s} V_{k}$ is the sum of the associative strengths of all the CSs occurring on that trial.

Consider now what happens if $A$ occurs but is not followed by the US. On these "extinction" trials, the association between $A$ and the US decreases according to an analogous formulation,

$$
\Delta V_{A}=\alpha_{A} \beta_{\downarrow}\left(0-\sum_{k \in s} V_{k}\right),
$$

where $\beta_{\downarrow}$ is the learning rate on trials when the US is absent. In general, $\beta_{\uparrow}$ is assumed 
to be larger than $\beta_{\downarrow}$, reflecting the fact that the presence of a US is more salient than its absence, but this is not critical for most predictions of the model (Rescorla \& Wagner, 1972).

Equations (1) and (2) of the Rescorla-Wagner model account for Kamin's blocking effect as follows. In phase $1, \mathrm{CS} A$ is conditioned to the US, and $V_{A}$ approaches $\lambda$. At the start of phase 2, the initial associative strength, $V_{B}$, of the CS $\mathbf{B}$ is assumed to be zero. The associative strength of the stimulus compound $A B$, therefore, is $\sum_{k \in s} V_{k}=V_{A}+$ $V_{B}=\lambda$. Intuitively, this implies that because the US is already perfectly predicted by $A$, the incremental learning accruing to the novel light stimulus, $\Delta V_{B}$, is predicted to be zero. Thus, prior learning to $A$ is expected to "block" later learning to $B$-just as was observed by Kamin (1969).

\section{THE CEREBELLUM AND CLASSICAL CONDITIONING}

There have been many attempts over the years to explain cerebellar function in motor learning, especially conditioning of the eyeblink response. The anatomy of the cerebellar cortex is remarkably uniform, with two separate input systems: the climbing fibers and the mossy fibers/parallel fibers that provide inputs from a variety of sensory modalities.

Figure 2 serves to summarize the overall empirical results to date from Thompson and colleagues. It is a much simplified version of a more comprehensive qualitative theory

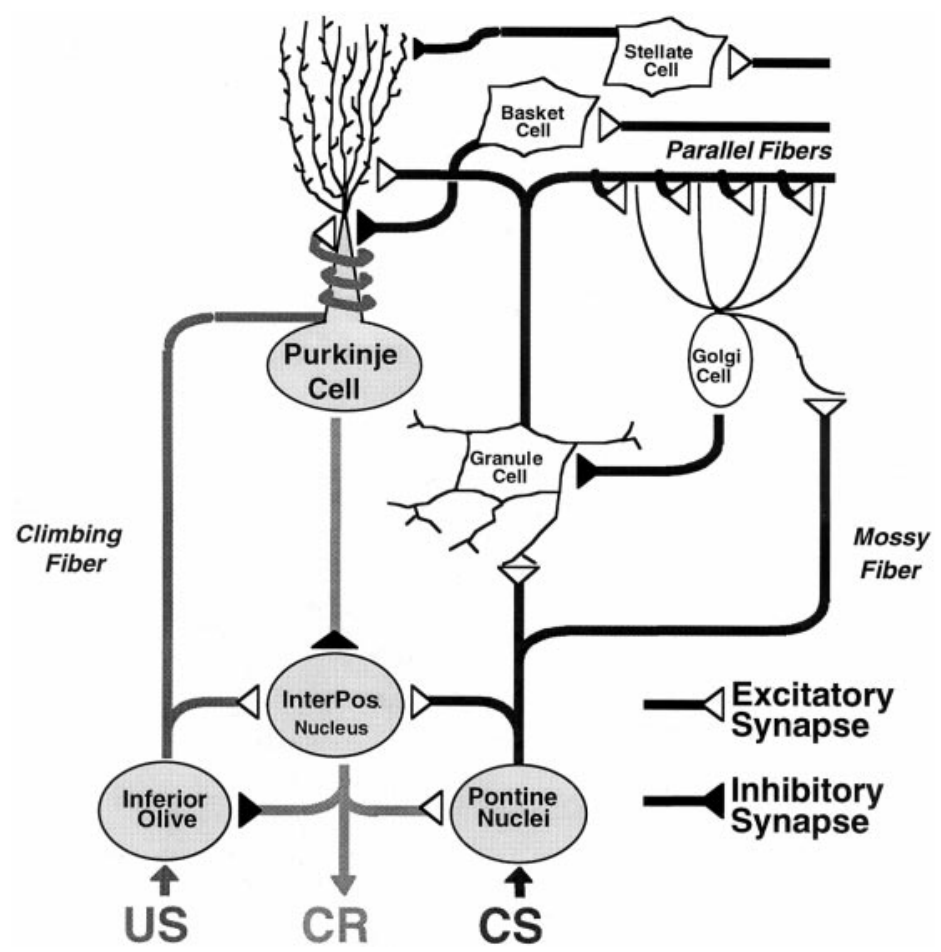

FIG. 2. Schematic of the cerebellar and brain stem circuitry thought to underlie eyeblink conditioning. The right pathway represents the CS input pathways via the mossy fiber and parallel fiber system. The left pathway represents the US input pathways via the climbing fiber system. The CS and US pathways converge on Purkinje cells and the interpositus nucleus. The middle pathway represents the CR output pathway and feedback loops onto the CS and US systems. 
of the role of the cerebellum in classical conditioning (see Thompson, 1986; Kim \& Thompson, 1997). As indicated in Fig. 2, there are two major projection systems to the cerebellum. The mossy fibers project from the pontine nuclei and other sources to the deep cerebellar nuclei and cerebellar cortex and convey, in the case of eyeblink conditioning, information about a tone or light CS. Information about the air puff or eye shock US in eyeblink conditioning projects to the inferior olive, which in turn projects to the deep cerebellar nuclei and cerebellar cortex via climbing fibers. The cerebellar cortex projects out through the deep cerebellar nuclei, specifically the interpositus nucleus in the case of eyeblink conditioning. The motor output target of the interpositus nucleus is the red nucleus, which in turn projects to motor nuclei in the brain stem (not shown in Fig. 2).

Anatomical tracing studies have demonstrated the connections between the inputs from the inferior olive and pontine nuclei to the Purkinje cells and interpositus nucleus (e.g., Brodal, 1981; Steinmetz \& Sengelaub, 1992; Tracey, Thompson, Krupa, \& Thompson, 1998). In addition, output from the interpositus to the red nucleus has been shown along with both the feedback connections onto the inferior olive and pontine nuclei (e.g., Steinmetz \& Sengelaub, 1992).

To determine the exact function of the CS and US inputs into the cerebellum, studies have been conducted that use electrical stimulation to simulate CS and US inputs, creating an eyeblink paradigm with no external sensory stimuli. Direct electrical stimulation of the lateral pontine nuclei can be substituted for a tone CS (Steinmetz, Rosen, Chapman, Lavond, \& Thompson, 1986), and stimulations of the dorsal accessory olive can be substituted for an air puff US (Mauk, Steinmetz, \& Thompson, 1986). It is even possible to have no external stimuli and condition rabbits with only paired presentations of pontine and olivary stimulation and still get conditioned eyeblink responding that is identical to that obtained via tone-air puff training (Steinmetz, Lavond, \& Thompson, 1989).

Lesion studies have sought to delineate the distinct roles of the cerebellar cortex and interpositus nucleus in acquisition and performance of conditioned eyeblinks. Lesions of the interpositus nucleus completely and permanently abolish previously learned conditioned responding (Steinmetz \& Sengelaub, 1992) and block acquisition in naive animals (McCormick \& Thompson, 1982), with no significant effects on performance of the reflex itself (Ivkovich, Lockard, \& Thompson, 1993). Lesions of the cerebellar cortex disrupt conditioning, but small-amplitude, poorly timed conditioned responses do slowly develop across training (Lavond et al., 1987; Woodruff-Pak, Lavond, Logan, Steinmetz, \& Thompson, 1993). Anterior lesions of cerebellar cortex have also been found to disrupt previous precisely timed conditioned responses (Logan, 1991; Perret, Ruiz, \& Mauk, 1993). Overall, it appears that the interpositus nucleus is responsible for the formation and execution of the conditioned response, whereas the cerebellar cortex is responsible for the correct timing of the conditioned response and controls the amplitude of the conditioned response.

In addition to the permanent lesions, key structures and pathways in the cerebellum have been reversibly inactivated using drugs and cooling. In well-trained animals, inactivation of the interpositus and overlying cortex of lobule HVI, of the red nucleus, or of the superior cerebellar peduncle (which conveys all output from the interpositus nucleus to descending and ascending systems) completely abolishes the expression of the CR. Inactivation of all these structures has no effect on the UR. When inactivation of the red nucleus or superior cerebellar peduncle occurs during training, the animals exhibit no CRs, but when subsequently tested without inactivation, they exhibit asymptotic CRs. This finding 
indicates that the animals learn the conditioned response but that its behavioral expression is blocked by the inactivation. Inactivation of the interpositus nucleus during training also results in no CRs; however, when tested without inactivation, animals still exhibit no CRs and subsequently learn as though they are naive (Clark \& Lavond, 1993; Clark, Zhang, \& Lavond, 1992; Krupa \& Thompson, 1995, 1997; Krupa, Thompson, \& Thompson, 1993; Ramirez, Nordholm, \& Thompson, 1997). This finding indicates that inactivation of the interpositus blocks not only expression but also acquisition of the CR. Electrophysiological recordings in the interpositus during inactivation of the red nucleus in trained animals show normal learning-related changes exhibiting the properties of the conditioned responses that are not being expressed, whereas inactivation of the interpositus nucleus abolishes both the behavioral CR and the learning-related unit activity in the red nucleus (Chapman, Steinmetz, Sears, \& Thompson, 1990; Clark \& Lavond, 1993). Consequently, the memory trace must be formed in the cerebellum.

Taken together, all of these lines of evidence provide strong support for the cerebellar model shown in Fig. 2. Simply put, the cerebellar cortex and interpositus receive CS tone information from the lateral pontine nuclei and receive US air puff information from the dorsal accessory olive. Convergence of the CS and US information in the cerebellum drives learning. The CR-related activity is transmitted to the brain stem motor nuclei along with positive feedback to the pontine nuclei and negative feedback to the inferior olive. This idea of a positive feedback loop from the cerebellum had been proposed as early as Blomfield and Marr (1970), who described how positive feedback could allow for temporal persistence of discharges in motor cortex. Houk, Singh, Fisher, and Barto (1990) implemented a cerebellar-red nucleus feedback system into a network model of the limb pre-motor network. The modules of their network are called adjustable pattern generators (APGs). This term refers to the ability of the module to generate an elemental burst of activity with adjustable intensity and duration. In general, in their model, Purkinje cells must become responsive to the sensory input that triggers the movement but must become unresponsive during the execution of the movement. This is accomplished by having Purkinje cells switch to a programming state in which Purkinje cell responsivity to the input is inhibited, allowing for positive feedback through the limb pre-motor network. Positive feedback through the limb pre-motor network switches the limb premotor network from an inactive to an active state and initiates the movement.

Houk et al. (1990) theorized that once the Purkinje cells switch to a particular firing state, they become refractory to further input until near the termination of the movement. A key assumption of this model is that the Purkinje cells are trained by the climbing fiber input to recognize the patterns of parallel fiber activity that indicates that the desired end point is about to be reached. This signal would activate Purkinje cells that were previously not responsive and allow them to fire, thereby terminating positive feedback through the pre-motor network.

This positive feedback loop was posited by Houk (1989) to be responsible for eyeblink conditioning. Specifically, Houk suggested that the excitatory activity in the interpositus nucleus is due not to direct sensory responses to the CS but rather to positive feedback in the recurrent network among the red nucleus, lateral reticular nucleus, and cerebellar interpositus nucleus. A weak CS input to the red nucleus needs to be fed back through this recurrent network to be amplified to the point where it is strong enough to drive a 
CR. The amplitude and duration of the CR are controlled by the inhibition of the activity within this loop by the Purkinje cells.

This APG model of the cerebellar brain stem role in classical conditioning suggests that the CS-US association is formed mainly outside of the cerebellum (in the red nucleus, lateral reticular nucleus, or even motor cortex). The role of the cerebellum is to determine the topography (i.e., amplitude and timing) of the CR.

Although recording studies of Purkinje cell firing patterns (Berthier \& Moore, 1986) and firing patterns in the red nucleus (Desmond \& Moore, 1991) support the APG model, there is some evidence from the temporary inactivation studies that does not. The reversible inactivation studies noted earlier report normal development of CR-related activity in the interpositus nucleus during red nucleus inactivation (Clark \& Lavond, 1993; Clark et al., 1992; Krupa \& Thompson, 1995, 1997; Krupa et al., 1993; Ramirez et al., 1997). If CRrelated activity develops normally in the cerebellum while the red nucleus is inactivated, then red nucleus-interpositus recurrencies cannot be responsible of the development of the CR.

However, one feature of the APG model that fits in nicely with the Thompson (1986) cerebellar model is that Purkinje cells are trained by the climbing fiber input to recognize the patterns of parallel fiber activity that indicates that the desired end point is about to be reached. Houk et al. (1990) did not specify what this pattern of activity from parallel fibers is. The Thompson (1986) model would suggest that this pattern of activity of parallel fiber is the efferent copy of the CR that is projected via the pontine nucleus back to the cerebellar cortex. McCormick, Lavond, and Thompson (1983) recorded in the LPN subsets of neurons that either show CS onset-related activity or CS onset-related activity along with CR-related activity. This CR-related activity late during the CS period would serve as the signal to the Purkinje cells that the desired movement (i.e., eyelid closure) is about to be reached.

\section{Functional Significance of Inhibitory Feedback for Error Correction Learning}

In our work on the essential neural circuit for classical conditioning of discrete behavioral responses (see Fig. 2), we have discovered a circuit-level mechanism that can account for the error-correcting property of the Rescorla-Wagner rule. It is an emergent property of the organization of the neural circuit itself rather that a specialized synaptic process.

We assume that the amount of associative strength added on a given CS-US trial is proportional to the amount of climbing fiber activation of the cerebellum on that trial. Thus, we have conceptualized the reinforcement strength on a given trial as the proportion of effective climbing fibers activated by the US. Empirical evidence to date is strongly consistent with this hypothesis. In electrophysiological recordings from Purkinje cells made prior to training, presentation of the US consistently evokes climbing fiber activity, which in turn evokes complex spikes from Purkinje cells. In well-trained animals, the US typically does not evoke complex spikes (Foy \& Thompson, 1986; Krupa, Weiss, \& Thompson, 1991). Therefore, a marked decrease in climbing fiber activation of Purkinje cells is evident as a result of learning.

Recent anatomical and physiological evidence also indicates the existence of an inhibitory pathway from the interpositus directly to the inferior olive (Hesslow \& Ivarsson, 1996; Nelson \& Mugnoini, 1987; Steinmetz \& Sengelaub, 1992), implying that CR output 
could weaken the ability of the US to activate climbing fibers. There also is an indirect pathway from the interpositus to the red nucleus to the inferior olive that could serve a similar function (Weiss, McCurdy, Houk, \& Gibson, 1985). Then, as the CR is increasingly learned, activation of the US pathway by the external US is increasingly attenuated (see also Donegan et al., 1989; Donegan \& Wagner, 1987). This change in US inputs could implement the supposition inherent in the Rescorla-Wagner rule that the amount of associative strength added on each trial decreases as the CR increases.

More direct evidence for this hypothesis has been obtained by recording neuronal activity in an area of the inferior olive known as the dorsal accessory olive (DAO) that is activated by the corneal air puff US (Sears \& Steinmetz, 1990). US-alone presentations consistently evoke a phasic increase in response of the DAO neurons. As the behavioral CR begins to develop, this US-evoked response in DAO neurons becomes markedly attenuated. In a well-trained animal, US onset-evoked activity may be completely absent in the DAO on trials in which the animal gives a CR. However, US-alone presentations still evoke the same onset response that the US evoked prior to training. This finding indicates that the $\mathrm{CR}$ does indeed inhibit inferior olive activity when exhibited during a paired trial.

\section{A COMPUTATIONAL MODEL OF CEREBELLAR ERROR CORRECTION LEARNING}

We have instantiated these ideas within a computational model that incorporates the key pathways of the circuit schematic in Fig. 2. As shown in Figs. 3A and 3B, we have organized the cerebellar connectivity shown in Fig. 2 so that all sensory inputs enter at the bottom of the circuit and progress upward to the CR outputs at the top of the figure. In developing this model, we have adopted a major simplification: representing the Purkinje and interpositus nucleus as a two-layer module with inputs that represent the CS, US, and recurrent CR information as shown in Fig. 3A. The connectionist model of conditioning based on these simplifications is shown in Fig. 3B. The input-layer nodes represent granule cells that receive all possible CSs and become active when that CS is present. This information, corresponding to parallel fiber projections from granule cells, projects to an internal layer representing Purkinje cells and an output node representing the interpositus nucleus. The output from this node is the behavioral CR. The internal-level input weights to the internal layer are random and fixed, whereas the weights to the output node are adaptive. The activity of the output node also has excitatory recurrent connections to the CS inputs along with inhibitory recurrent connections to the US inputs. Within our circuit model, we have implemented this negative feedback loop with a nonmodifiable synapse of value -1 between the output node (interpositus) and the inferior olive. The functional role of this inhibitory feedback is to compute the error signal measured by the inferior olive's activity. The inferior olive's activity is governed by the following equation:

$$
I O=(U S-C R)
$$

where $I O$ is the activity of the inferior olive, $U S$ is the US input, and $C R$ is the output of the output node (interpositus). Thus, the inferior olive's output is projected to the 
(A). Simplified

\section{Cerebellar Circuits}

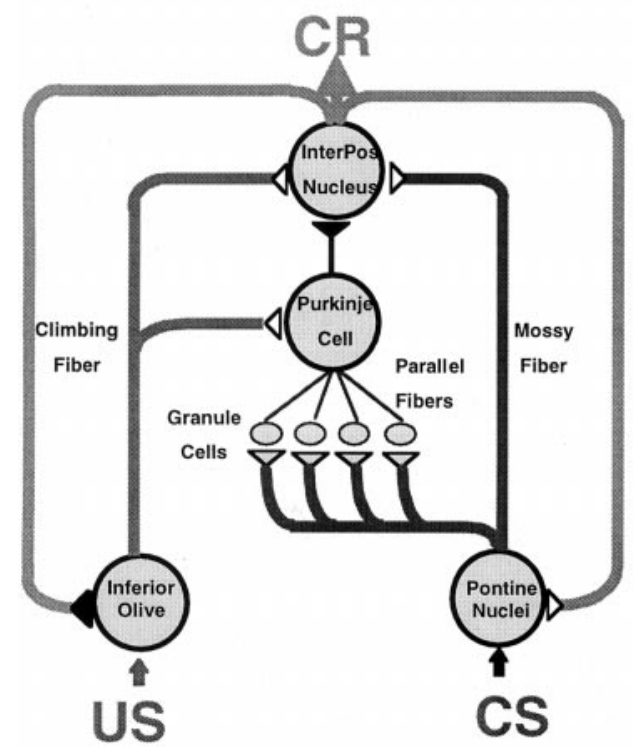

(B). Connectionist Model of Conditioning

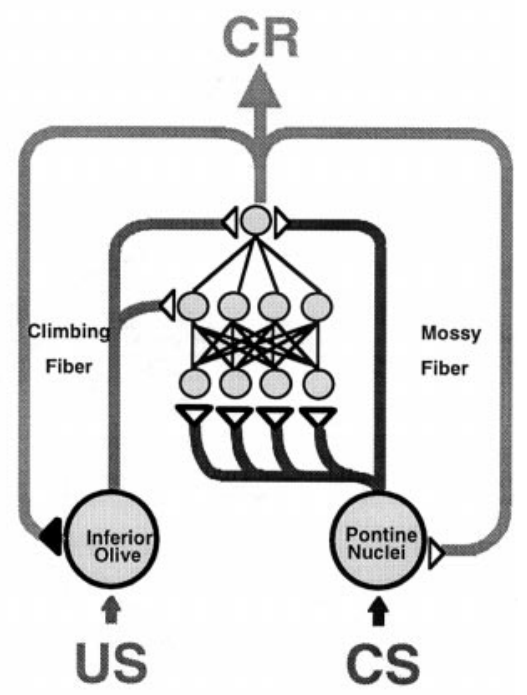

FIG. 3. Simplifications that have been made to adapt the cerebellar anatomy into the connectionist architecture for the development of the current computational model of the cerebellum. (A) Cerebellar circuit diagram that has been simplified from the cerebellar circuitry shown in Fig. 2. The circuit has been organized so that sensory inputs enter at the bottom of the figure and inputs flow upward to the CR outputs at the top of the figure. Furthermore, the cerebellar cortex has been simplified into a layer that represents the granule cells, a node that represents the Purkinje cells, and a node that represents the interpositus nucleus. (B) Connectionist model of conditioning that has been designed based on the simplified cerebellar circuit in panel A. The input layer represents the granule cells. The internal layer represents the Purkinje cells. The output node represents the interpositus nucleus.

internal layer and output node as an "error signal" that is analogous to the term $\left(\lambda-\Sigma_{j} C S_{j} w_{j}\right)$ in Eq. (1) of the Rescorla-Wagner equation.

Implicit in the above discussion is the assumption that activity in the inferior olive can represent both errors of omission and errors of commission. Errors of commission occur, for example, on extinction trials and during conditioned inhibition training when a response is made but no US occurs. Without a baseline level of activity in the inferior olive, the inhibitory effect of a response on an inactive inferior olive (e.g., an extinction trial) would have no effect. Without the ability to represent negative errors of commission, the system has no way of representing a trial on which associative weights should decrease.

We can accommodate the need for representing negative errors by assuming that the inferior olive has a positive baseline level of firing when no error has occurred. Depressing the inferior olive below this baseline represents negative error. In this way, the inferior olive term would reflect not the absolute activity level of the inferior olive but rather its activity level relative to baseline activity. There is some empirical evidence to support this assumption; the spontaneous discharge rate of inferior olivary neurons is about 2 to 4 spikes per second in the awake-behaving animal (Ito, 1984). As noted earlier, a naive animal shows US-evoked activity in the dorsal accessory olive. However, as learning occurs, this US-evoked activity in the dorsal accessory olive decreases to near zero on 
paired CS-US trials as the animal learns to predict the US from the CS and reliably exhibit a CR.

Our simplified circuit model of the cerebellum predicts that when a well-trained animal gives a $\mathrm{CR}$ to the $\mathrm{CS}$ on CS-alone trials, the spontaneous response rate of dorsal accessory olivary neurons should decrease to below baseline levels during the CS period. This prediction follows from the neurobiological model on the assumption that increased activity of the interpositus nucleus results in increased direct inhibition of olivary neurons.

\section{Simulation of Acquisition of Conditioned Response}

The simplest form of eyeblink classical conditioning is delay conditioning. In delay conditioning, a CS is repeatedly paired with a US, with some specified delay between CS and US onset (the interstimulus interval [ISI]), so that the CS and US coterminate. With the exception of trace conditioning (defined later), all simulations reported are based on delay conditioning.

The model is a discrete time model. This means that at each cycle the model can modify its synapses only once, as opposed to real-time biological systems that continuously modify synapses. In these simulations, we divided the trial into 10 discrete cycles or time intervals. At this stage in theory development, we make no direct mapping between the duration of a cycle and an actual time interval. A simulation of simple delay conditioning (see Fig. 4) shows the results of training the model on a delay paradigm in which the CS is on (input activation $=1$ ) for cycles 4 through 8 inclusive, whereas the US is on only for cycle 8 (i.e., an ISI of 4 time cycles).

The model is able to simulate a variety of features of normal delay conditioning. It

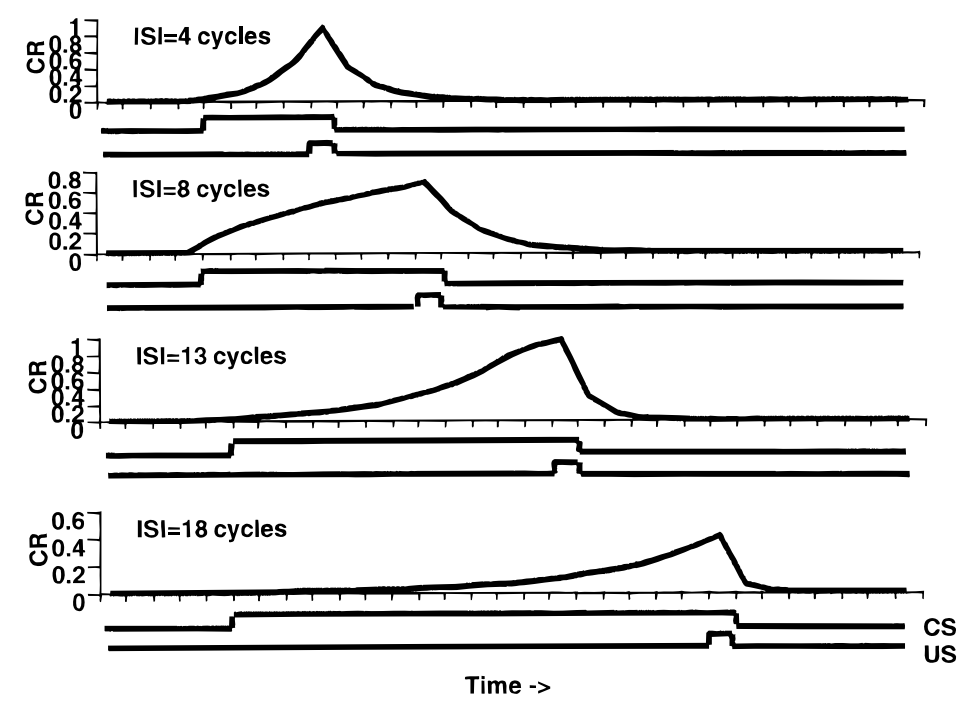

FIG. 4. Simulation results from our cerebellar model that correctly mimic CR characteristics for eyeblink conditioning with well-timed CR topographies across a variety of ISIs (4-18 time cycles). In each case, the peak CR response occurs at about the time of US onset, as is seen in behavioral literature. In addition, note that in the case of the responses to the ISI of 18 time cycles, twice as many trials were required for proper timing as compared to the shorter ISIs. This simulation agrees with the behavioral literature showing that the longer the ISI, the slower the learning. 
shows CR development into a well-timed response in which the CR peak occurs at about the time of US onset. The timing of the CR peak is correct over a range of ISI lengths from 4 to 18 time cycles, as shown in Fig. 4. In animals (Gormezano et al., 1983), the different ISI lengths result in different learning rates in that the fastest acquisition occurs for about 250 to $400 \mathrm{~ms}$ ISI and is slower for each successively longer ISI. This finding is demonstrated in our modeling simulations because it takes twice as many trials to achieve well-timed CRs with an 18-time-cycle ISI as with a 4-time-cycle ISI. In the model, this happens because the only change in net input from cycle to cycle is the changing CR feedback. The network must learn a set of weights such that the CR remains low for the first part of the ISI and grows rapidly thereafter. With short ISI training, it is appropriate to exhibit CRs much sooner after CS onset; therefore, this task is learned more quickly. The increased complexity of the long ISI task not only increases learning time but also results in a broader CR, consistent with experimental data (Gormezano et al., 1983). We theorize that the well-timed CR is due to recurrent feedback of the CR (e.g., to the pontine nuclei). If this feedback loop to the CS input pathway is disabled, then the cerebellar model is unable to properly time the CR to peak at about the US onset.

\section{Simulating Complex Learning and the Role of Informational Variable}

The preceding simulations have illustrated how the cerebellar circuit model generates a wide range of behaviors appropriate to the acquisition of the conditioned eyeblink response in rabbits. The model can also account for numerous learning phenomena believed to involve more subtle aspects of stimulus selection. We discuss several representative examples below including conditioned inhibition, positive and negative patterning, and blocking.

Conditioned inhibition is a phenomenon where paired presentations of a CS $A$ and the US are intermixed with nonreinforced compound presentations of $A$ and a second cue, CS $B$ ( $A+, A B-$ training). The Rescorla-Wagner rule predicts that strong associative strength accrues to $A$, whereas strong negative associative strength accrues to $B$ and limits the associative strength that can accrue to $A$. In the second phase, learning is compared between $B$ and a novel cue, $C$. Therefore, in the second phase of training, responding to $B$ is slower because $B$ has a negative associative strength as compared to the novel cue, $C$, which has no associative strength. The conditioned inhibition effect has been demonstrated in rabbit eyeblink conditioning (Mahoney, Kwaterski, \& Moore, 1975; Marchant \& Moore, 1974; Solomon, 1977). As shown in Fig. 5A, the model quickly learns to generate a strong $\mathrm{CR}$ to $A+$ trials but not to $A B$ - trials in phase 1 . In phase 2 , conditioned inhibition is exhibited by the fact that learning to $B+$ is slower than learning to the novel cue $C$, as demonstrated by an increased number of trials necessary to reach a criterion of $80 \%$ CRs (see Fig. 5B).

Another issue for theories of conditioned inhibition is whether or not the presentation of the inhibiting cue alone ( $B$ in the above discussion) results in extinction of the inhibitory properties of this cue. Remember that in tests of extinction of conditioned inhibition, presentations of $A+$ are intermixed with presentations of $A B$ in phase 1. Extinction trials consisting of $B$ alone are presented in phase 2. Test trials of $A+$ and $A B-$ are presented in phase 3. If extinction has not occurred, then $B$ should continue to be just as inhibitory to responding to $A$ despite the intervening $B$-alone extinction trials. The simulation results 
A

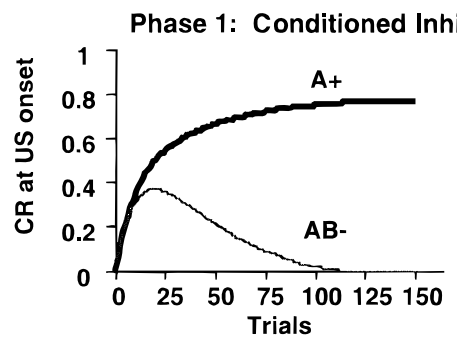

B

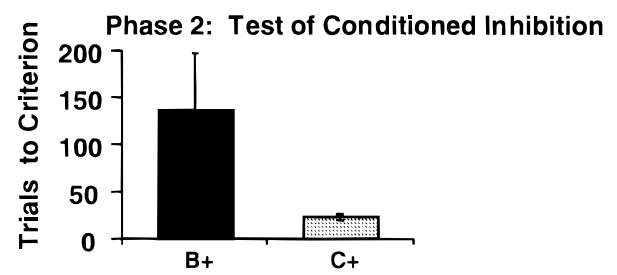

FIG. 5. Simulation results from our cerebellar model for conditioned inhibition. (A) Learning curves for phase 1 of conditioned inhibition. In phase 1 , there is strong responding on $A+$ trials (blue line) and some initial responding on $A B-$ trials (red line) that is inhibited later in training. (B) Slower learning in phase 2 on $B+$ trials as compared to $C+$ trials as represented as trials to reach a criterion of more than $80 \%$ CRs.

from our cerebellar model do not show an extinction of conditioned inhibition following unreinforced presentation of the inhibitor. This makes intuitive sense. If a cue predicts the absence of a US and there is no US following the cue, then we would naturally expect that the cue would retain its inhibitory value. The simulation results from our cerebellar error-correcting model show the strong response to $A$ and equally weak responses to $A B$ both before extinction trials (Fig. 6A) and following $2000 \mathrm{~B}$--alone extinction trials (Fig. 6B).

Interestingly, the issue of what happens during cue-alone presentation of a previously conditioned inhibitor is one of the most problematic aspects of the Rescorla-Wagner model. The Rescorla-Wagner model erroneously predicts extinction of conditioned inhibition in these circumstances, and experimental studies have shown that in animals the conditioned inhibitor does not extinguish with cue-alone presentation (Zimmer-Hart \& Rescorla, 1974). As shown in Fig. 6C, conditioned inhibition is shown in the Rescorla-Wagner model by little responding to $B$ immediately following $A B$ trials. Following 2000 trials of $B$ alone, the Rescorla-Wagner rule predicts that the conditioned inhibition effect is extinguished, as demonstrated by equivalent responding to $A+$ and $A B-$ trials as shown in Fig. 6D. Thus, although our model can be seen as a mapping of the Rescorla-Wagner model onto circuit-level mechanisms, there clearly is a subtle difference between our cerebellar model and the original Rescorla-Wagner model theory; the cerebellar model shows the behaviorally appropriate lack of extinction of conditioned inhibition.

The difference in results between the Rescorla-Wagner rule and our cerebellar model reflects differences in what values are actually subtracted for the error correction calculation. In the Rescorla-Wagner rule, the error comparison during phase $2 B$-alone extinction trials is between $\lambda$ and $V_{B}$. At the start of $B$-alone extinction training, $\lambda$ is zero and the $V_{B}$ has a negative value. This discrepancy drives learning to that the value of $V_{B}$ approaches zero. When $A B$ - test trials are now presented, $V_{B}$ equals zero and $V_{A}$ still has a positive value. Therefore, both $A+$ and $A B-$ trials now elicit CRs following the $B$-alone extinction 
trials, whereas previously $A B$ - trials resulted in little responding. In our cerebellar model, the error comparison made by the inferior olive is not between $\lambda$ and $V_{B}$ but rather between the values of the US and CR. During $B$-alone extinction trials, there is no US and no CR, and both values are zero; therefore, there is no error to drive any learning during the $B$ alone extinction trials. Because of this lack of learning, the extinction training has no effect on subsequent $A B$ test trials, and conditioned inhibition is exhibited just as before the $B$-alone extinction trials.

Another conditioning task for which the cerebellar network model can account is blocking. As described earlier, blocking is the phenomenon where a neutral CS $A$ is first repeatedly paired with the US ( $A+$ training) so that the animal comes to expect the US given $A$ (see Table 1). In the second phase of training, animals receive trials with stimulus compound of previously trained $A$ and a novel CS $B$, paired with the same US $(A B+)$. A later test to the individual CSs shows a continued high level of responding to $A$ but little responding to $B$. Apparently, the prior conditioning to $A$ blocks the learning to $B$. By contrast, control animals that receive training to a neutral CS $C$ in phase 1 followed by $A B+$ trials show appreciably more responding when tested with $B$. Marchant and Moore

\section{Cerebellar Error-Correction Model Prediction: No Extinction of CI}

A Phase 2: Test of CI

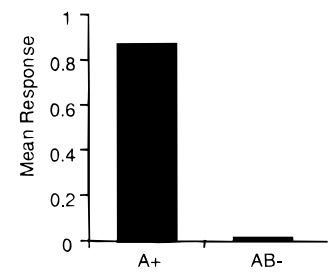

B After 2000 B- Trials: Test of CI

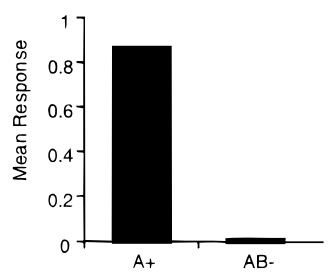

\section{Rescorla-Wagner Model Prediction: Exinction of CI}

\section{Phase 2: Test of CI}

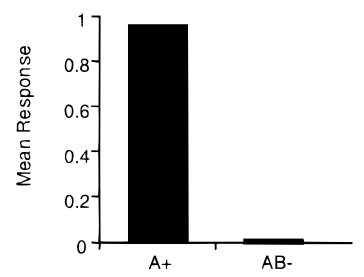

D After 2000 B- Trials: Test of CI

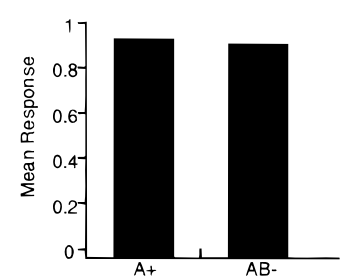

FIG. 6. Simulation results from our cerebellar model that correctly predict a lack of extinction of conditioned inhibition following $2000 \mathrm{~B}-$ trials (A, B). These results are compared to the incorrect Rescorla-Wagner prediction of elimination of conditioned inhibition following this manipulation (C, D). (A) Results from our cerebellar model in which there is strong responding on $A+$ trials and virtually no responding to $A B-$ trials at the end of conditioned inhibition training. (B) Responding following $2000 \mathrm{~B}$ - extinction trials in which the inhibitory effect of $B$ has not been extinguished. (C) Results expected by the Rescorla-Wagner rule in which there is strong responding to $A+$ trials and virtually no responding to $A B-$ trials at the end of conditioned inhibition training. (D) Incorrect extinction of conditioned inhibition following $B$-alone trials as predicted by the Rescorla-Wagner rule. 
(1973), Solomon (1977), and Kehoe (1981) demonstrated this blocking phenomenon in rabbit eyeblink conditioning, whereas Martin and Levey (1991) demonstrated blocking with human eyeblink conditioning. As expected, the cerebellar model shows robust blocking in these training conditions. Figure 7 shows the slower learning to $B+$ training following $A+$ and $A B+$ training as compared to the control group, which was previously trained on $C+$ and $A B+$.

Two predictions about the neural mechanisms of blocking can be made from the cerebellar model. First, in well-trained animals, the US consistently evokes complex spikes in Purkinje cells on US-alone trials. However, on CS-US trials where the animal generates a CR, the US did not evoke complex spikes. The model predicts that this lack of Purkinje complex spiking is due, we think, to CR feedback from the interpositus, inhibiting dorsal accessory olive activity by way of the inhibitory neurotransmitter gamma-aminobutyric acid (GABA). If the feedback path from the interpositus to the DAO is inactivated, then the US should evoke Purkinje cell complex spikes even if the CR is generated. To test this prediction, Kim et al. (1998) implanted cannulas in the DAO to infuse the GABA antagonist picrotoxin in animals well trained to respond to a tone CS. Picrotoxin has the effect of blocking the inhibitory action of GABA on neurons. After picrotoxin infusion, these same Purkinje neurons consistently exhibited complex spikes to US onset on paired trials where the animal performed CRs. It would seem that inactivation of the feedback inhibitory projection from interpositus to the DAO in well-trained animals now permits the US to fully activate the DAO-climbing fiber system (the reinforcing or teaching input) just as in naive animals.

Second, it follows from the network model and the result just described that inactivation of the GABA-ergic projection to the DAO during the compound phase of training in the blocking paradigm should prevent blocking. Kim et al. (1998) tested this prediction with implanted cannulas in the DAO that infused the GABA antagonist picrotoxin in animals well trained to respond to a tone CS. Rabbits in a blocking group were given initial training to the tone $(T)$ and were then injected with the vehicle solution of artificial cerebrospinal fluid (ACSF) and trained to the compound of tone and light (TL). These rabbits were markedly impaired in subsequent acquisition to the light $(L)$, compared to

Phase $3(\mathrm{~B}+)$ learning speed:

Test for Blocking

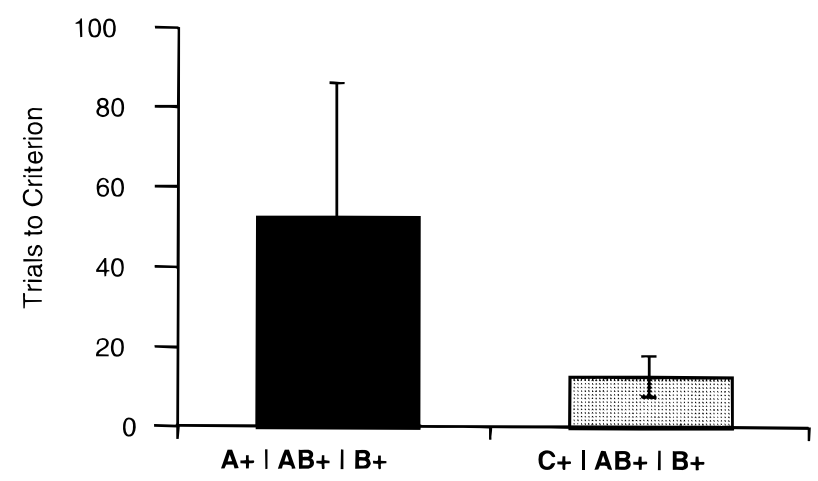

FIG. 7. Simulation results for the blocking phenomena as the difference in trials to criterion on $B+\operatorname{training}$ following either $A+\mid A B+$ or $C+\mid A B+$ training. The blocking effect is demonstrated in that previous training to $A+\mid A B+$ significantly slows subsequent learning to $B+$ as compared to previous training to $C+\mid A B+$. 


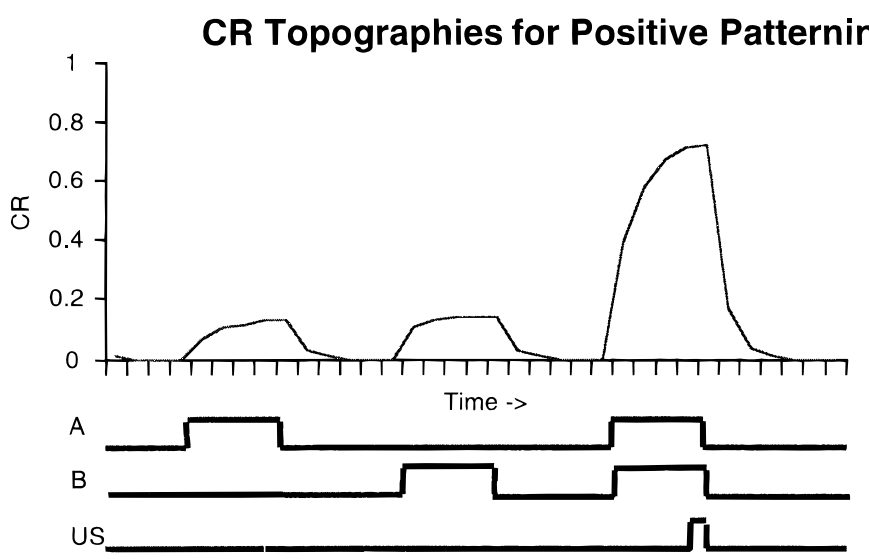

FIG. 8. Simulation results for the positive patterning phenomena, as shown by CR topography on all three trial types. There is a strong response on the $A B+$ trials, whereas the responding on $A+$ and $B+$ trials is inhibited.

the control group not given initial training to the tone $(T)$ and trained only to the compound tone-light $(T L)$.

By contrast, rabbits first trained to the tone $(T)$ that then received the GABA antagonist picrotoxin during compound training to the tone and light compound (TL) showed no blocking at all; they subsequently learned to the light just as did the control group not given initial training to the tone $(T)$. Therefore, inactivation of GABA inhibition in the inferior olive "blocks" blocking, as predicted by the cerebellar network model.

Another type of learning task that has been explained to a limited degree by the Rescorla-Wagner rule and the cerebellar network model includes configural learning tasks such as positive and negative patterning. A configural task is one in which a response to a compound cue is different from the response to the individual components. It was proposed by Rudy and Sutherland (1995) that an intact hippocampal region is necessary to learn this type of configural task.

Positive patterning is a phenomenon in which $A$ and $B$ presented individually are not paired with the US, but the compound $A B$ is paired with the US. Therefore, the animal learns to respond to the compound but to not respond to the individual cues. The RescorlaWagner model predicts that the associative strengths of the individual cues $A$ and $B$ are not strong enough to elicit a $\mathrm{CR}$, whereas the summed associative strengths of the compound $A B$ are strong enough to reliably elicit a CR. The Rescorla-Wagner model predicts that if the associative strength of $A B=1$, then $A$ alone plus $B$ alone should equal 1 (e.g., $A=0.5$ and $B=0.5$ ), but this is higher than the response to $A$ or $B$ seen in animals given positive patterning. Note that this is true only if one assumes no configural nodes in the Rescorla-Wagner model. Our cerebellar model embeds the Rescorla-Wagner rule, with the difference being a hidden layer that can form configural associations.

Bellingham, Gillette-Bellingham, and Kehoe (1985) and Kehoe and Schreurs (1986) demonstrated the positive patterning effect by training rabbits with presentations of a tone and light paired with the US along with an equal number of the nonreinforced presentations of the tone and light individually. The model is able to simulate this result. As shown by CR topographies in Fig. 8, responding is strong to the compound cue $A B$, whereas responding to the individual cues $A$ and $B$ is inhibited.

Negative patterning is the opposite phenomenon to positive patterning. In negative 
patterning, CS $A$ and CS $B$ are each individually paired with the US, whereas the compound presentations of $A B$ are not reinforced. The animal, therefore, must learn to respond when either CS appears alone but not to respond when the compound is presented. The Rescorla-Wagner rule can account for negative patterning only if additional assumptions are made (Gluck \& Bower, 1988). The additional assumptions are the hidden layer, as in our cerebellar model.

Bellingham et al. (1985) demonstrated the negative patterning phenomenon by training rabbits that either a tone and light alone predicted the US air puff, whereas the compound tone-light presentation did not. Differentiation between the individual and compound presentations in the negative patterning paradigm was much slower to develop than in positive patterning. Early during training, there was more responding to the compound cue than to the individual cues. Eventually, however, rabbits learned to respond to the cues instead of the compound.

Although the model easily simulates positive patterning, the model has some trouble with negative patterning. Examples of CR topography from a simulation that correctly solved the negative patterning task (labeled "Solver" in Fig. 9A) and a simulation that did not correctly solve the negative patterning task (labeled "Non-Solver" in Fig. 9B). This bimodal result may be indicative of the complex nature of the task and the problems that have been reported in obtaining the negative patterning result in animals. Negative patterning has been considered a form of a "configural" task that requires sensitivity to CS-CS relationships that many have argued depends not just on the cerebellum but also on the hippocampus (Sutherland \& Rudy, 1989). As such, it is not surprising that a model of only the cerebellar contribution to learning would have difficulty in reproducing a

\section{CR Topographies for Negative Patterning}

A

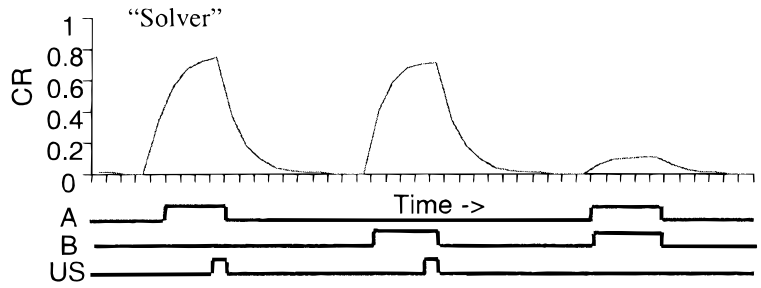

B

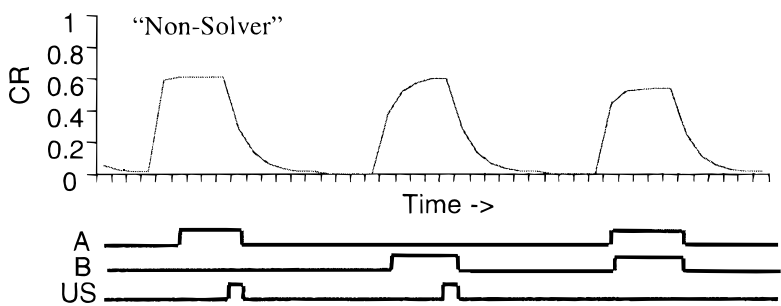

FIG. 9. Simulation results for the negative patterning phenomena. (A) Conditioned response topography for the three trial types $(A+, B+, A B-)$ for a simulation that correctly solved the task labeled a "Solver." This simulation result correctly solved the task by responding to $A+$ and $B+$ trials while inhibiting responding toe $A B+$ trials. (B) Conditioned response topography for the three trial types $(A+, B+, A B-)$ for a simulation that one that failed to solve the task labeled a "Non-Solver." This simulation result failed to solve the task due to responding indiscriminately to all three trial types. 
behavior seen most clearly in animals with intact hippocampal functioning. In the next section, we explore this idea further.

\section{IMPLICATIONS FOR MODELS OF HIPPOCAMPAL FUNCTION IN CONDITIONING}

Motor learning such as eyeblink conditioning can engage higher brain structures such as the hippocampus. In delay eyeblink conditioning, animals with nonselective hippocampal region lesions (Schmaltz \& Theios, 1972) and selective hippocampal lesions (Shohamy et al., 2000) can learn and retain CRs relatively normally. However, nonselective hippocampal region lesions markedly impair more complex forms of conditioning such as trace conditioning (Moyer, Deyo, \& Disterhoft, 1990; Solomon, Vander Schaaf, Thompson, \& Weisz, 1986), discrimination reversal learning (Berger \& Orr, 1983), latent inhibition (Solomon \& Moore, 1975), and learned irrelevance (Allen, Chelius, \& Gluck, 1998). Furthermore, hippocampal lesions made immediately after training abolish the trace eyeblink CR, whereas lesions made a month after training do not impair CR retention (Kim, Clark, \& Thompson, 1995). This anterograde amnesia and time-limited retrograde amnesia are the hallmarks of medial-temporal lobe/hippocampal-dependent declarative memory processes in humans (Squire, 1992; Squire \& Zola-Morgan, 1991), suggesting that trace eyeblink conditioning may be a useful elementary animal model of human declarative memory (Clark \& Squire, 1998).

Apparently, there are learning pathways in the hippocampus and cerebellum that ordinarily operate in parallel. Some behaviors are critically dependent on the hippocampus and are impaired when the hippocampus is damaged. For other behaviors, cerebellar mediation may suffice. Because the current cerebellar model exists in the absence of a model of hippocampal function, we expect to see the same range of behaviors from this cerebellar model as in animals that have undergone hippocampal removal.

\section{Correspondence with Cerebellar Circuit Model}

Consistent with the data on animals with hippocampal lesions (Moyer et al., 1990), our cerebellar model is unable to learn with trace conditioning (i.e., conditioning in which the CS terminates prior to US onset and a memory "trace" of the CS must be maintained while no stimuli are present) or with very long ISIs in a delay conditioning paradigm. The model has considerable difficulty in learning tasks that require sensitivity to configurations. The model suggests that learning these tasks is due to the higher order filtering of the input lines to the internal layer and output node, but not nearly enough to account for the complete differentiation between stimulus components and compounds observed in asymptotic negative patterning. This is consistent with data showing that lesioning of the hippocampus and associated structures can impair configural learning (Rudy \& Sutherland, 1995; Sutherland \& Rudy, 1989).

Hippocampal-lesioned animals show impairments, compared to intact animals, in several learning paradigms in which preexposure to one or more CSs affects the ability of the animals to form associations in later conditioning. Consistent with the behavioral data (Port \& Patterson, 1984), the cerebellar model fails to exhibit sensory preconditioning (McFarland, Kostas, \& Drew, 1978) and latent inhibition (Solomon \& Moore, 1975). 
For sensorimotor learning tasks, such as eyeblink conditioning, our model and the Kim et al. (1998) data argue that the contribution of US modulation to stimulus selection in blocking arises from intrinsic circuits of the cerebellum via an error-correcting inhibitory feedback pathway that is functionally analogous to the process proposed by Rescorla and Wagner (1972). This leaves open, however, the possibility that additional CS modulation influences on stimulus selection and blocking may be mediated by other brain structures such as the hippocampus. Although blocking in other preparations, such as emotional (fear) conditioning, may involve important contributions from both CS and US modulation, Kim et al.'s (1998) data argue strongly that cerebellar-mediated US modulation is sufficient to mediate blocking in rabbit eyeblink conditioning.

\section{Correspondence with Cerebellar-Hippocampal Model of Gluck and Myers}

The Gluck and Myers (1993) hippocampal model proposed how the hippocampus might modulate the cerebellum during eyeblink conditioning. The hippocampal region is modeled as a network that learns to reproduce its inputs at the output layer, as shown in Fig. 10. Because the middle node layer is narrower than the input and output layers, it is necessary to form a stimulus representation in the middle layer that compresses redundant information while preserving predictive information. The stimulus representations formed in the hippocampal region network are transmitted as a training signal to a long-term memory module. In the case of eyeblink conditioning, it is assumed that the long-term memory component is the cerebellum. This module is functionally similar to our cerebellar model. These stimulus representations are mapped by the cerebellum to an output that is interpreted as the behavioral response.

The Gluck-Myers cortico-hippocampal model is capable of learning phenomena that are

A

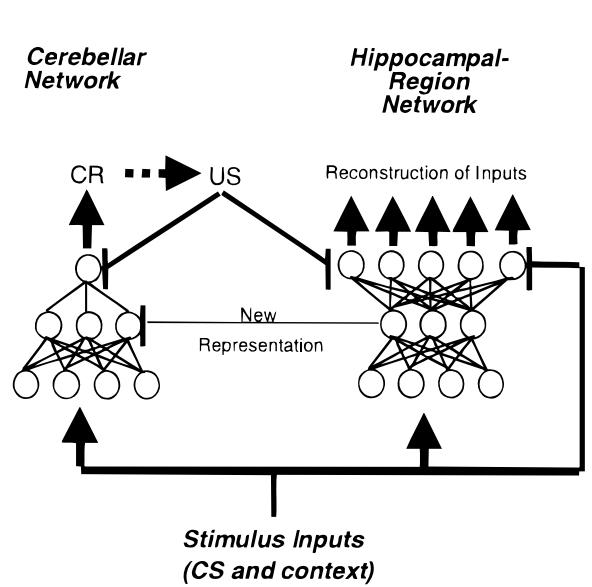

B

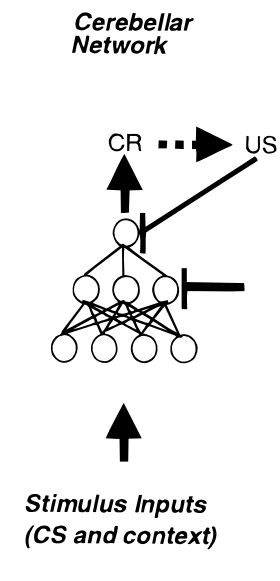

FIG. 10. Gluck and Myers (1993) model of hippocampal modulation of cerebellar learning in eyeblink conditioning. (A) Intact model consisting of both the hippocampal region network and the cerebellar network. The representations formed by the hippocampal module are sent to the internal layer (Purkinje cells) of the cerebellar module. (B) In the lesioned model, the hippocampal region network has been removed, leaving the cerebellar network and its stimulus inputs. 
A. Thompson (1986) Circuit
C. Gluck \& Myers (1993) Trial-Level Lesioned (Cereb) Model
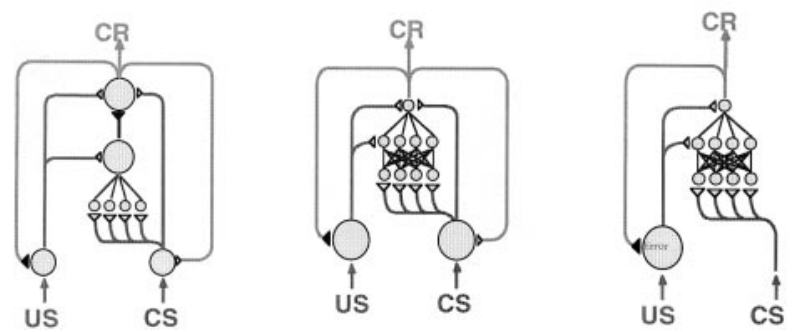

FIG. 11. Equivalency among (A) the simplified cerebellar circuit (Thompson, 1986), (B) the current cerebellar model, and (C) the Gluck and Myers (1993) hippocampal-lesioned (cerebellum alone) trial-level model.

not possible in our current error-correcting cerebellar model. The bias toward redundancy compression can account for learning phenomena such as latent inhibition, learned irrelevance, sensory preconditioning, and compound preconditioning. The bias toward predictive differentiation can account for learning phenomena such as discrimination reversal learning, easy-hard transfer, and stimulus generalization. With the hippocampal region module removed and the hippocampus effectively lesioned, the remaining long-term memory component can be thought of as an isolated cerebellar model with no hippocampal modulation, as shown in Fig. 11C.

Overall, the current error correction cerebellar model and the Gluck-Myers corticohippocampal model can be viewed as continuations of our ongoing work to investigate the roles of various brain structures, specifically the cerebellum and hippocampus, in classical eyeblink conditioning as schematized in Fig. 11.

\section{GENERAL DISCUSSION}

We have presented a computational model of the cerebellar circuitry believed to mediate eyeblink conditioning and other discrete sensorimotor learning tasks. Our primary focus has been to review the evidence for a negative feedback circuit that implements an error correction learning procedure, much like the Rescorla-Wagner model of conditioning. In addition to accounting for a range of conditioning behaviors previously localized to the cerebellum, this cerebellar model correctly predicts behavioral deficits associated with lesions of the hippocampal formation (e.g., trace conditioning, latent inhibition).

The current real-time model has come about from a series of previous trial-level instantiations of the idea of error correction in the cerebellum (Bartha, Thompson, \& Gluck, 1991; Donegan et al., 1989; Gluck et al., 1990, 1994). This real-time precision is due to the recurrent connection between the interpositus output and the pontine nuclei. Through this pathway, the cerebellum is able to learn not only the CS-US association but also the CR-US association and, thus, inhibit the CR until the time of the US onset.

This article has focused on the ability of the error-correcting cerebellar circuitry to simulate basic eyeblink conditioning characteristics along with more complex learning phenomena that have previously been explained by the Rescorla-Wagner rule. The Rescorla-Wagner model and the concept of error correction not only are applicable to simple 
forms of learning such as classical conditioning but also have been applied to more cognitive tasks in humans along with being a foundation for connectionist models of human learning (Gluck \& Bower, 1988).

The Rescorla-Wagner model and basic error correction cannot account for all learning phenomena such as latent inhibition. These limitations can be viewed as suggesting that these behavioral paradigms require brain structures and learning mechanisms other than error correction alone. In particular, the hippocampus has been implicated as playing a modulatory and necessary role in some learning phenomena that are not accounted for by the Rescorla-Wagner rule.

Previous work with a computational model of the hippocampus (Gluck \& Myers, 1993) has shown that the addition of stimulus selection abilities of the hippocampus along with the error correction of the cerebellum can account for a wider range of conditioning phenomena than can the Rescorla-Wagner model alone. These findings indicate that the limitations of the Rescorla-Wagner model are not due to a failure of the theory; rather, there exist multiple types of learning mechanisms that are activated by different types of tasks.

Additional support for the idea that neural circuits are capable of instantiating error correction learning comes from the paradigm of fear conditioning and the work of Mike Fanselow and colleagues at the University of California, Los Angeles (Fanselow, 1998). In fear conditioning, a neutral CS is paired with an electric shock. The CS acquires the ability to elicit several fear-related behaviors including an endogenous opioid-mediated analgesia (Fanselow, 1984). Fear conditioning requires that the US be painful to be effective, and therefore, the analgesic state elicited by the CS would reduce the reinforcing strength of the shock. In the case of blocking, initial training with a tone and shock would bring about this analgesic state in response to the tone. When a tone and light are subsequently paired with the shock, the shock would be ineffective in producing a fear response to the light because the shock is no longer painful by virtue of the analgesic effect of the previously conditioned tone. In this way, the analgesic effect would provide negative feedback on the acquisition of fear conditioning. This feedback loop can be interpreted as calculating the Rescorla-Wagner rule by way of the tone's analgesic effect reducing the strength of the shock until there is no response to the shock and, therefore, no reinforcement for learning to respond to the light.

Fanselow (1998) mapped this fear conditioning analgesic effect onto the amygdala and its related structures. The tone CS information reaches the amygdala by way of the thalamus, whereas the shock US information reaches the amygdala by way of the dorsal horn. The amygdala is the site of CS-US-related synaptic plasticity and provides negative feedback to the dorsal horn US projection system by way of the endogenous opioid release from the ventral periaqueductal gray that creates the analgesic state that blocks the painful nature of the shock. This amygdala circuit for fear conditioning is directly comparable to our cerebellar model of eyeblink conditioning. In both cases, there is a negative feedback pathway that blocks the US information from effectively reaching the site of CS-US convergence in well-trained animals. In both cases, this negative feedback pathway can be blocked pharmacologically. As with the Kim et al. (1998) eyeblink experiment in which the negative feedback from the cerebellum to the inferior olive is blocked by injection of picrotoxin that eliminated the blocking effect, an analogous study has been done in fear conditioning. When the negative feedback opioid pathway for fear conditioning 
is blocked by administration of the opioid antagonist naloxone, the blocking effect is attenuated (Fanselow \& Bolles, 1979).

Overall, evidence from the cerebellum and the amygdala point to the existence of negative feedback loops in the neural circuitry thought to underlie two different classical conditioning paradigms that are capable of calculating the Rescorla-Wagner rule. It is likely that the Rescorla-Wagner rule not only is applicable to our work with the cerebellum and eyeblink conditioning and Fanselow's work with the amygdala and fear conditioning but may also be involved in other forms of associative learning for which the underlying neural circuitry is not fully understood.

\section{Future Directions}

It will be critical in future studies to seek to understand how the hippocampus is able to modulate the cerebellar circuits in a real-time fashion so that the aggregate behavior of both systems behaves like the connectionist network described here. Another important line of research for future development will be to extend the model to more accurately reflect cerebellar circuitry and to account for the individual neuronal contributions of the cerebellar cortex and interpositus such as the granule, stellate, and Golgiss cells in cerebellar cortex and to determine the role of excitatory and inhibitory neurons within the interpositus that are the basis for the feedback pathways to the inferior olive and pontine nuclei. Further work also needs to be done to test the relative roles of the hippocampus and cerebellum in learning phenomena such as blocking in a variety of learning paradigms other than eyeblink conditioning.

\section{APPENDIX}

The cerebellar model consists of four input nodes fully connected to 20 hidden nodes; all of the input and hidden nodes are connected to a single output node. The activation of this output node is the behavioral response or CR. On each training cycle, three input nodes represent the presence (1) or absence (0) of three CSs: $A, B$, and $C$. The fourth input node is a feedback projection from the output node; its activation is the behavioral response $C R$ from the previous training cycle. The $C R$ is initialized to 0 at the start of the simulation run. All input-output (IO) and hidden-output weights are initialized to 0.0; input-hidden weights are initialized from the uniform distribution $(-0.3,+0.3)$.

Each trial is divided into cycles, each representing about $50 \mathrm{~ms}$. Each trial contains 30 cycles unless otherwise noted below. All CSs are 0 at the beginning of a trial. At least 1 $\mathrm{CS}$ arrives at some prescheduled cycle in the trial and lasts for a predetermined number of cycles. If the US is present, then it lasts for exactly 1 cycle and coterminates with the CS. If no CSs are present during a cycle, then the network's only nonzero input is the CR feedback. The network is given 1000 initialization trials, with all CSs and US set to 0.0.

On each cycle, each hidden node $j$ computes its activation, $A_{j}$, according to

$$
A_{j}=\sum_{i} I_{i} w_{i j}
$$

where $i$ ranges over the four inputs ( $A, B, C$, and $C R$ ), $I_{i}$ is the activation of the corresponding input node, and $w_{i j}$ is the weight from $C S_{i}$ to node $j$. Activation is clipped at 0.0 and 1.0. 
The output node calculates its activation according to the same rule except that $i$ ranges over the input and hidden nodes.

Next, network weights are updated. The output error is computed as

$$
\text { Error }=I O=(U S-C R)
$$

where $U S$ is 1 if the US is present and 0 otherwise, and $C R$ is the activation of the output node. Both the input-output and hidden-output nodes update weights as

$$
\Delta w_{i j}=\beta(\text { Error }) A_{i},
$$

where learning rate $\beta=0.04$ if the US is present and 0.004 otherwise.

In the picrotoxin simulations of Fig. $11 \mathrm{~B}$, the $\mathrm{CR}$ feedback to $\mathrm{IO}$ is assumed to be disabled, and so the error computation of Eq. (A2) is replaced by

$$
\text { Error }=I O=U S \text {. }
$$

All results shown are averaged over 10 simulation runs. Criterion performance (as in Fig. 5B) is defined as 10 consecutive trials during which the network output is greater than 0.80 on all US-present cycles and less than 0.20 throughout presentation of all CSs not associated with the US.

Acquisition. As shown in Fig. 4, onset of CS A occurs at cycle 4, and US onset occurs $4,8,13$, or 18 cycles later. Both $A$ and US coterminate 1 cycle later. Figure 4 shows output node activation for each cycle during a trial after 1000 trials $($ ISI $=4)$, after 5000 trials (ISIs $=8,13)$, and after 10,000 trials (ISI $=18$ ).

Conditioned inhibition. For the first 5000 trials, the network is given $A$-US and $A B$-no US training. Within each 30 -cycle trial, $A$ onset occurs at cycle 4 , US onset occurs at cycle 8 , and both $A$ and US coterminate at cycle 9; $A$ and $B$ onset then occurs at cycle 19 with offset at cycle 24 and no US. Figure 5A shows the output node activation at cycle $8(A+)$ and cycle $23(A B-)$. Following this training (Fig. 5B), there are 500 trials alternating training to $B$ and a new CS: $C$. $C$ onset occurs at trial 4 , whereas $B$ onset occurs at trial 19; in both cases, US onset occurs 4 trials later and then CS and US coterminate.

Figure 6A shows the model responding after the first phase of $A+, A B-$ training; the network responds strongly to $A$ alone and weakly to $A B$. Following 2000 trials of $B-$ alone ( $B$ onset at cycle 19 , offset at cycle 24 , no US), the network continues to respond strongly to $A$ and weakly to $A B$ (Fig. 6B).

Positive and negative patterning. For positive patterning, trials consist of 40 cycles. $A$ is presented from cycles 4 through $8, B$ from cycles 19 through 23 , and $A B$ from cycles 34 through 38 ; the US is on cycle 38 . For negative patterning, the procedure is the same as for positive patterning except that the US is presented on cycles 8 and 23 .

Blocking. Phase 1 consists of 4000 20-cycle trials with $A$ onset occurring at cycle 4, US onset at cycle 8, and offset of both $A$ and US at cycle 9. Phase 2 consists of 500 trials that are similar except that $B$ is presented simultaneously with $A$. Finally, phase 3 consists of 500 trials in which $B$ is presented alone and coterminates with the US. Phase 3 
performance is compared to a control condition that received $C$-US training in phase 1 but similar phase 2 and phase 3 treatment.

\section{REFERENCES}

Allen, M. T., Chelius, L., \& Gluck, M. A. (1998). Selective entorhinal ibotenic lesions disrupt the learned irrelevance pre-exposure effect in the classically conditioned rabbit eyeblink response paradigm conditioning. Society for Neuroscience Abstracts, 24.

Anderson, B. J., \& Steinmetz, J. E. (1994). Cerebellar and brainstem circuits involved in classical eyeblink conditioning. Reviews in Neuroscience, 5(3), 1-23.

Bartha, G. T., Thompson, R. F., \& Gluck, M. A. (1991). Sensorimotor learning and the cerebellum. In M. Arbib \& J. Ewert (Eds.), Visual structures and integrated functions. Berlin: Springer-Verlag.

Bellingham, W. P., Gillette-Bellingham, K., \& Kehoe, E. J. (1985). Summation and configuration in patterning schedules with the rat and rabbit. Animal Learning and Behavior, 13, 152-164.

Berger, T., \& Orr, W. (1983). Hippocampectomy selectively disrupts discrimination reversal learning of the rabbit nictitating membrane response. Behavioral Brain Research, 8, 49-68.

Berthier, N. E., \& Moore, J. W. (1986). Cerebellar Purkinje cell activity related to the classically conditioned nictitating membrane response. Experimental Brain Research, 63, 341-350.

Berthier, N. E., Singh, S. P., Barto, A. G., \& Houk, J. C. (1993). Distributed representations of limb motor programs in arrays of adjustable pattern generators, Journal of Cognitive Neuroscience, 5, 56-78.

Blomfield \& Marr. (1970). How the cerebellum may be used. Nature, 227, 1224-1228.

Brodal, A. (1981). Neurological anatomy, New York: Oxford Univ. Press.

Chapman, P. F., Steinmetz, J. E., Sears, L. L., \& Thompson, R. F. (1990). Effects of lidocaine injection in the interpositus nucleus and red nucleus on conditioned behavioral and neuronal responses. Brain Research, 537, 140-156.

Clark, R. E., \& Squire, L. R. (1998). Classical conditioning and brain systems: The role of awareness. Science, 280, 77-81.

Clark, R. E., \& Lavond, D. G. (1993). Reversible lesions of the red nucleus during acquisition and retention of a classically conditioned behavior in rabbit. Behavioral Neuroscience, 107, 264-270.

Clark, R. E., Zhang, A. A., \& Lavond, D. G. (1992). Reversible lesions of the cerebellar interpositus nucleus during acquisition and retention of a classically conditioned behavior. Behavioral Neuroscience, 106, 879-888.

Desmond, J. E., \& Moore, J. W. (1991). Altering the synchrony of stimulus trace processes: Tests of a neuralnetwork model. Biological Cybernetics, 65, 161-169.

Donegan, N. H., Gluck, M. A., \& Thompson, R. F. (1989). Integrating behavioral and biological models of conditioning. Psychology of Learning and Motivation, 3, 109-156.

Donegan, N. H., \& Wagner, A. R. (1987). Conditioned diminution and facilitation of the UR: A sometimes opponent-process interpretation. In I. Gormezano \& W. F. Prokasy (Eds.), Classical conditioning (3rd ed.). Hillsdale, NJ: Erlbaum.

Fanselow, M. S. (1984). Shock-induced analgesia on the formalin test: Effects of shock severity, naloxone, hypophysectomy, and associative variables. Behavioral Neuroscience, 98, 79-95.

Fanselow, M. S. (1998). Pavlovian conditioning, negative feedback, and blocking: Mechanisms that regulate association formation. Neuron, 20, 625-627.

Fanselow, M. S., \& Bolles, R. C. (1979). Naloxone and shock-elicited freezing in the rat. Journal of Comparative and Physiological Psychology, 93, 36-44.

Foy, M. R., \& Thompson, R. F. (1986). Single unit analysis of Purkinje cell discharge in classically conditioned and untrained rabbits. Neuroscience Abstracts, 12, 518.

Gluck, M. A., \& Bower, G. H. (1988). From conditioning to category learning: An adaptive network model. Journal of Experimental Psychology: General, 117, 227-247.

Gluck, M., \& Myers, C. (1993). Hippocampal mediation of stimulus representation: A computational theory." Hippocampus, 3, 491-516. 
Gluck, M., Myers, C., \& Thompson, R. F. (1994). A computational model of the cerebellum and motor-reflex conditioning. In Zornetzer, Davis, Lau, \& McKenna (Eds.), An introduction to neural and electronic networks (pp. 91-98). New York: Academic Press.

Gluck, M. A., Reifsnider, E. S., \& Thompson, R. F. (1990). Adaptive signal processing and the cerebellum: Models of classical conditioning and VOR adaptation. In M. A. Gluck \& D. E. Rumelhart (Eds.), Neuroscience and connectionist theory (pp. 131-185). Hillsdale, NJ: Erlbaum.

Gormezano, I., Kehoe, E. J., \& Marshall, B. S. (1983). Twenty years of classical conditioning research with the rabbit. Progress in Psychobiology and Physiological Psychology, 10, 197-275.

Hebb, D. O. (1949). The organization of behavior: A neuropsychological theory. New York: Wiley.

Hesslow, G., \& Ivarsson, M. (1996). Inhibition of the inferior olive during conditioned responses in the decerebrate ferret. Experimental Brain Research, 110, 36-46.

Houk, J. C. (1989). Cooperative control of limb movements by the motor cortex, brainstem, and cerebellum. In R. M. J. Cotterill (Ed.), Models of brain function. Cambridge, UK: Cambridge Univ. Press.

Houk, J. C., Keifer, J., \& Barto, A. G. (1993). Distributed motor commands in the limb premotor network. Trends in Neuroscience, 16, 27-33.

Houk J. C., Singh, S. P., Fisher, C., \& Barto, A. G. (1990). In W. T. Miller, R. S. Sutton, \& P. J. Werbos (Eds.), Network for neural control (pp. 301-348). Cambridge, MA: MIT Press.

Hull, C. (1943). Principles of behavior. New York: Appleton-Century-Crofts.

Ito, M. (1984). The cerebellum and neural control. New York: Raven.

Ivkovich, D., Lockard, J. M., \& Thompson, R. F. (1993). Interpositus lesion abolition of the eyeblink CR is not due to effects on performance. Behavioral Neuroscience, 107, 530-532.

Kamin, L. (1969). Predictability, surprise, attention, and conditioning. In B. Campbell \& R. Church (Eds.), Punishment and aversive behavior (pp. 279-296). New York: Appleton-Century-Crofts.

Kehoe, E. (1981). Stimulus selection and combination in classical conditioning with the rabbit. In I. Gormezano, W. Prokasy, \& R. Thompson (Eds.), Classical conditioning (Vol. 1). Hillsdale, NJ: Erlbaum.

Kehoe, E. J., \& Schreurs, B. G. (1986). Compound-component differentiation as a function of CS-US interval and CS duration in the rabbit's conditioned nictitating membrane response. Animal Learning \& Behavior, 14, 144-154.

Kim, J. J., Clark, R. E., \& Thompson, R. F. (1995). Hippocampectomy impairs the memory of recently, but not remotely, acquired trace eyeblink conditioned responses. Behavioral Neuroscience, 109, 195-203.

Kim, J., Krupa, D., \& Thompson, R. F. (1998). Inhibitory cerebello-olivary projections and blocking effect in classical conditioning. Science, 27, 570-573.

Kim, J. J., \& Thompson, R. F. (1997). Cerebellar circuits and synaptic mechanisms involved in classical eyeblink conditioning. Trends in Neuroscience, 20, 177-181.

Krupa, D. J., Thompson, J. K., \& Thompson, R. F. (1993). Localization of a memory trace in the mammalian brain. Science, 260, 989-999.

Krupa, D. J., \& Thompson, R. F. (1995). Inactivation of the superior cerebellar peduncle blocks expression but not acquisition of the rabbit's classically conditioned eyeblink response. Proceedings of the National Academy of Science, 92, 5097-5101.

Krupa, D. J., \& Thompson, R. F. (1997). Reversible inactivation of the cerebellar interpositus nucleus completely prevents acquisition of the classically conditioned eyeblink response. Learning and Memory, 3, 545-556.

Krupa, D. J., Weiss, C., \& Thompson, R. F. (1991). Air puff evoked Purkinje cell complex spike activity is diminished during conditioned responses in eyeblink conditioned rabbits. Society for Neuroscience Abstracts, 17, 322 .

Logan, C. G. (1991). Cerebellar cortical involvement in excitatory and inhibitory classical conditioning. Doctoral dissertation, Stanford University.

Mahoney, W. J., Kwaterski, S. E., \& Moore, J. W. (1975). Conditioned inhibition of the rabbit nictitating membrane response as a function of CS-UCS interval. Bulletin of the Psychonomic Society, 5, 77-179.

Marchant, H., \& Moore, J. (1973). Blocking of the rabbit's conditioned nictitating membrane response in Kamin's two-stage paradigm. Journal of Experimental Psychology, 101, 155-158. 
Marchant, H., \& Moore, J. (1974). Below-zero conditioned inhibition of the rabbit's nictitating membrane response. Journal of Experimental Psychology, 102, 350-352.

Mauk, M. D., Steinmetz, J. E., \& Thompson, R. F. (1986). Classical conditioning using stimulation of the inferior olive as the unconditioned stimulus. Proceedings of the National Academy of Sciences (USA), 83, 5349-5353.

McCormick, D., Lavond, D., \& Thompson, R. F. (1983). Neuronal responses of the rabbit brainstem during performance of the classically conditioned nictitating membrane (NM)/eyelid response. Brain Research, 271, 73-88

McCormick, D., \& Thompson, R. (1982). Locus coeruleus lesions and resistance to extinction of a classically conditioned response: Involvement of the neocortex and hippocampus. Brain Research, 245, 239-249.

McFarland, D. J., Kostas, J., \& Drew, W. G. (1978). Dorsal hippocampal lesions: Effects of preconditioning CS exposure to flavor aversion. Behavioral Biology, 22, 398-404.

Miller, R., Barnet, R., \& Grahame, N. (1995). Assessment of the Rescorla-Wagner model. Psychological Bulletin, 117, 363-386.

Moyer, J., Deyo, R., \& Disterhoft, J. F. (1990). Hippocampectomy disrupts trace eye-blink conditioning in rabbits. Behavioral Neuroscience, 104, 243-252.

Nelson, B., \& Mugnoini, E. (1987). GABAergic innervation of the inferior olivary complex and experimental evidence for its origin. In P. Strata (Ed.), The olivocerebellar system in motor control. New York: Springer-Verlag.

Perret, S. P., Ruiz, B. P., \& Mauk, M. D. (1993). Cerebellar cortex lesions disrupt learning-dependent timing of conditioned eyelid responses. Journal of Neuroscience, 13, 1708-1718.

Port, R. L., \& Patterson, M. M. (1984). Fimbria lesions and sensory preconditioning. Behavioral Neuroscience, 98, 584-589.

Ramirez, O. A., Nordholm, A. F., \& Thompson, R. F. (1997). The conditioned eyeblink response: A role for the GABA-B receptor. Pharmacology, Biochemistry, and Behavior, 58, 127-132.

Rescorla, R. (1968). Probability of shock in the presence and absence of CS in fear conditioning. Journal of Comparative and Physiological Psychology, 66, 1-5.

Rescorla, R., \& Wagner, A. (1972). A theory of Pavlovian conditioning: Variations in the effectiveness of reinforcement and non-reinforcement. In A. Black \& W. Prokasy (Eds.), Classical conditioning II: Current research and theory (pp. 64-99). New York: Appleton-Century-Crofts.

Rudy, J., \& Sutherland, R. (1995). Configural association theory and the hippocampal formation: An appraisal and reconfiguration. Hippocampus, 5, 375-398.

Rumelhart, D., Hinton, G., \& Williams, R. (1986). Learning internal representations by error propagation. In D. Rumelhart \& J. McClelland (Eds.), Parallel distributed processing: Explorations in the microstructure of cognition (Vol. 1, pp. 318-362). Cambridge, MA: MIT Press.

Schmaltz, L., \& Theios, J. (1972). Acquisition and extinction of a classically conditioned response in hippocampectomized rabbits (Oryctolagus cuniculus). Journal of Comparative and Physiological Psychology, 79, $328-333$.

Sears, L., \& Steinmetz, J. (1990). Acquisition of classically conditioned-related activity in the hippocampus is affected by lesions of the cerebellar interpositus nucleus. Behavioral Neuroscience, 104, 681-692.

Solomon, P. (1977). Role of the hippocampus in blocking and conditioned inhibition of the rabbit's nictitating membrane. Journal of Comparative and Physiological Psychology, 91, 407-417.

Solomon, P., \& Moore, J. (1975). Latent inhibition and stimulus generalization of the classically conditioned nictitating membrane response in rabbits (Oryctolagus cuniculus) following dorsal hippocampal ablation. Journal of Comparative and Physiological Psychology, 89, 1192-1203.

Solomon, P. R., Vander Schaaf, E. R., Thompson, R. F., \& Weisz, D. J. (1986). Hippocampus and trace conditioning of the rabbit's classically conditioned nictitating membrane response. Behavioral Neuroscience, 100, 729-744.

Spence, K. W. (1956). Behavior theory and conditioning. New Haven, CT: Yale Univ. Press.

Squire, L. R. (1992). Declarative and nondeclarative memory: Multiple brain systems supporting learning and memory. Journal of Cognitive Neuroscience, 4, 232-242. 
Squire, L. R., \& Zola-Morgan, S. (1991). The medial temporal lobe memory system. Science, 233, 1380-1386.

Steinmetz, J. E., Lavond, D. G., \& Thompson, R. F. (1989). Classical conditioning in rabbits using pontine nucleus stimulation as a conditioned stimulus and inferior olive stimulation as an unconditioned stimulus. Synapse, 3, 225-233.

Steinmetz, J. E., Rosen, D. J., Chapman, P. F., Lavond, D. G., \& Thompson, R. F. (1986). Classical conditioning of the rabbit eyelid response with a mossy fiber stimulation CS (Part 1). Behavioral Neuroscience, 100, 878-887.

Steinmetz, J. E., \& Sengelaub, D. R. (1992). Possible conditioned stimulus pathway for classical eyelid conditioning in rabbits: Anatomical evidence for direct projections from the pontine nuclei to the cerebellar interpositus nucleus. Behavioral \& Neural Biology, 57, 103-115.

Steinmetz, J. E., \& Thompson, R. F. (1991). Brain substrates of aversive classical conditioning. In Neurobiology of learning emotion and affect (pp. 97-120). New York: Raven.

Sutherland, R., \& Rudy, J. (1989). Configural association theory: The role of the hippocampal formation in learning, memory, and amnesia. Psychobiology, 17, 129-144.

Sutton, R. S., \& Barto, A. G. (1981). Toward a modern theory of adaptive networks: Expectation and prediction. Psychological Review, 88, 135-170.

Thompson, R. F. (1986). The neurobiology of learning and memory. Science, 233, 941-947.

Tracey, J., Thompson, J. K., Krupa, D. J., \& Thompson, R. F. (1998). Evidence of plasticity in the pontocerebellar CS pathway during classical conditioning of the eyeblink response in the rabbit. Behavioral Neuroscience, 112, 267-285.

Wagner, A. R. (1969). Stimulus selection and modified continuity theory. In G. Bower \& J. Spence (Eds.), The psychology of learning and motivation (Vol. 3). New York: Academic Press.

Wagner, A. R., \& Rescorla, R. (1972). Inhibition in Pavlovian conditioning: Application of a theory. In R. A. Boakes \& M. S. Halliday (Eds.), Inhibition and learning. New York: Academic Press.

Walkenbach, J., \& Haddad, N. F. (1980). The Rescorla-Wagner theory of conditioning: A review of the literature. Psychological Record, 30, 497-509.

Weiss, C., McCurdy, M. L., Houk, J. C., \& Gibson, A. R. (1985). Anatomy and physiology of dorsal column afferents to forelimb dorsal accessory olive. Society for Neuroscience Abstracts, 11, 182.

Widrow, B., \& Hoff, M. (1960). Adaptive switching circuits. Institute of Radio Engineers, Western Electronic Show and Convention Record, 4, 96-104.

Woodruff-Pak, D. S. (1999). New directions for a classical paradigm: Human eyeblink conditioning. Psychological Science, 10, 1-3.

Woodruff-Pak, D., Lavond, D., Logan, C. G., Steinmetz, J. E., \& Thompson, R. F. (1993). Cerebellar cortical lesions and reacquisition in classical conditioning of the nictitating membrane response in rabbits. Brain Research, 608, 67-77.

Zimmer-Hart, C., \& Rescorla, R. (1974). Extinction of Pavlovian conditioned inhibition. Journal of Comparative and Physiological Psychology, 86, 837-845. 